\title{
VOLATILIZAÇÃO DE N-NH 3 NA CULTURA DE MILHO: II. AVALIAÇÃO DE FONTES SÓLIDAS E FLUIDAS EM SISTEMA DE PLANTIO DIRETO E CONVENCIONAL ${ }^{(1)}$
}

\author{
W.A.R. LARA CABEZAS ${ }^{(2)}$, G.H. KORNDORFER ${ }^{(3)} \&$ S.A. MOTTA ${ }^{(4)}$
}

\begin{abstract}
RESUMO
Foram desenvolvidos dois experimentos em campo, em sistema de plantio direto (SPD) sobre cobertura de aveia-preta, em latossolo vermelho-escuro, distrófico, argiloso, e em sistema de plantio convencional (SPC), após cultivo de soja, em latossolo vermelho-amarelo distrófico arenoso, no Centro de Pesquisa NovartisSeeds e na Fazenda Sta. Teresinha, Uberlândia (MG) respectivamente. 0 estudo objetivou avaliar as perdas por volatilização de $\mathrm{N}-\mathrm{NH}_{3}$ da cobertura nitrogenada na cultura de mi lho com cerca de $100 \mathrm{~kg} \mathrm{ha}^{-1}$ de $\mathrm{N}$, de cinco fontes nitrogenadas em ambos os sistemas de plantio. As fontes nitrogenadas - sulfato de amônio, nitrato de amônio, uréia e duas soluções nitrogenadas constituídas de uréia + nitrato de amônio (uran) e uréia + nitrato de amônio + sulfato de amônio (sulfuran) - foram aplicadas na superfície e incorporadas no meio da entrelinha. Após a aplicação da cobertura, instalaram-se, ao acaso, três coletores do ti po semi-aberto estático, por tratamento, sendo efetuadas seis amostragens de $\mathrm{N}-\mathrm{NH}_{3}$ volatilizado, em intervalos de quatro a cinco dias. No SPD, as perdas acumuladas de $\mathbf{N}-\mathrm{NH}_{3}$ provenientes das fontes uréia, uran e sulfuran aplicadas na superfície foram, respectivamente, de 78,0; 37,2 e 26,9\% do $\mathbf{N}$ aplicado. No SPC, as perdas mais significativas foram de uréia $(30,7 \%)$ e uran $(9,7 \%)$. O nitrato de amônio e o sulfato de amônio apresentaram perdas inferiores a $15,0 \%$ do $\mathbf{N}$ aplicado à superfície. A correlação das perdas por volatilizacão de N$\mathrm{NH}_{3}$ e a produtividade dos dois experimentos mostraram um ajuste linear negativo, de tal forma que no SPD houve uma queda de produção de $13,3 \mathrm{~kg}$ de grãos e no SPC, de 11,8 kg de grãos para cada quilograma de $\mathbf{N}$ volatilizado.
\end{abstract}

Termos de indexação: plantio direto, plantio convencional, volatilização de amônia, adubos nitrogenados sólidos, adubos nitrogenados fluidos, milho.

\section{SUMMARY: $\mathrm{NH}_{3}-\mathrm{N}$ VOLATILIZATION IN CORN CROP: II - EVALUATION OF SOLID AND FLUID SOURCES UNDER NO-TILLAGE AND CONVENTIONAL SYSTEMS}

Two field experiments were carried out with corn, using no-tillage system (NTS) over oat residues, in a clay dark-red latosol at the Centro dePesquisa Novartis - Seeds, in U berlândia, State of Minas Gerais, Brazil , and conventional tillage system over soybean resi dues (CTS), in

\footnotetext{
(1) Trabalho apresentado no I Congresso de Plantio Direto, realizado em Ponta Grossa (PR), em 18-22 de março de 1996. Recebido para publicação em maio de 1996 e aprovado em junho de 1997.

(2) Pesquisador Visitante Estrangeiro, Departamento de Agronomia, Universidade Federal de Uberlândia, Caixa Postal 593, CEP 38406-067 U berlândia (MG).

(3) Professor Titular, Departamento deAgronomia, U niversidade Federal de U berlândia, Caixa Postal 593, CE P $38406-067$ U berlândia (MG).

(4) Técnico Agrícola, Centro de Pesquisas Novartis-Seeds, Rodovia BR-452, km 142, U berlândia (MG).
} 


\begin{abstract}
a sandy red-yel low latosol at the Santa Terezi nha farm, U berlândia. Theobjective of this study was to evaluate the $\mathrm{NH}_{3}-\mathrm{N}$ volatilized from nitrogen sources, sidedressed applications, of approximately $100 \mathrm{~kg} \mathrm{ha-1}$, applied in the middle of the rows. Five nitrogen sources [urea, ammoni um nitrate, ammonium sul phate, urea + ammonium nitrate soluti on (uran) and urea +ammonium nitrate + ammonium sul phatesoluti on (sul phuran)] and two forms of appl ication: surfaceand incorporated $(5-7 \mathrm{~cm})$ wereused in both crop systems. After thenitrogen sidedressing application, threesemi-open static col lectors of $\mathrm{NH}_{3}-\mathrm{N}$ wereinstalled by treatment. Six samples were taken at intervals of four to five days. In the NTS, the accumulative $\mathrm{NH}_{3}-\mathrm{N}$ Iosses from urea, uran and sul phuran were, respectively, 75.5, 39.2 and $25.5 \%$ of thesurface applied N. The most significant losses in the CTS occurred with urea (30.7\%) and uran (9.7\%). Ammonium sulphate and ammonium nitrate showed $\mathrm{NH}_{3}-\mathrm{N}$ losses lower than $15.0 \%$. A negativelinear regression was found between relative corn yield and $\mathrm{NH}_{3}-\mathrm{N}$ losses. The data showed that $7.9 \mathrm{~kg} \mathrm{ha}-1$ of grains were lost per kilogram of vol atilized nitrogen.
\end{abstract}

Index terms: no-tillage system, conventional system, $\mathrm{NH}_{3}-\mathrm{N}$ vol atilization, nitrogen fertilizers, fluid fertilizers, corn.

\section{INTRODUÇÃO}

A adubação nitrogenada em cobertura, na cultura de milho, é feita, principalmente, utilizando-se uréia na superfície, tanto no sistema de plantio direto (SPD) como no convencional (SPC). Entre os mecanismos de transformação do N-uréia aplicado ao solo, a vol atilização deamônia éum dos que contribuem para a baixa recuperação de $\mathrm{N}$ pela cultura (Ernst \& Massey, 1960; Bouwmeester et al., 1985). A quantificação dessas per das em condições de campo é fundamental para concluir sobre a conveniência de continuar com essa prática ou optar por um manejo alternativo. Volk (1959) registrou perdas de 29,3, 11,5 e $0,3 \%$ do $\mathrm{N}$ aplicado na forma de uréia, uran e nitrato de amônio respectivamente, à superfície do solo cultivado com pastagens. Eckert et al. (1986), em SPD de milho, obtiveram produtividades de 10,5, 9,9 e 9,9 t ha-1 de grãos quando cultivado sobre pal hada de soja, e de 10,4, 8,5 e 8,2 t ha-1 em palhada de milho, com a aplicação de amônia anidra injetada no solo, uréia e uran superficial respectivamente. Mengel et al. (1982), comparando a aplicação superficial e incorporada a $20 \mathrm{~cm}$ de profundidade de amônia anidra euran, verificaram maior rendimento de grãos de milho e teor foliar de N, quando as fontes foram incorporadas, tanto em SPD como em SPC. Timmons \& Baker (1992) mostraram que a utilização de um injetor pontual ("point injector"), para a incorporação do uran na cobertura nitrogenada de milho, foi mais eficiente quea incor poração com haste e aplicação em banda superficial. Resultados de setelocais (SPD), em Missouri (EUA), onde o milho foi cultivado após soja, mostraram produções decrescentes de grãos com aplicação de nitrato de amônio na superfície, uran incor porado ena superfície respectivamente (Stecker, 1995). Segundo Varsa et al. (1995), a uréia e o uran podem sofrer grandes perdas por volatilização quando colocados na superfície do solo e em contato com resíduos. $\mathrm{Na}$ hidrólise enzimática dessas fontes, se o $\mathrm{NH}_{4}{ }^{+}$não é absorvido pelo solo será perdido para a atmosfera. A cobertura fornecida pela palhada de milho (relação $\mathrm{C} / \mathrm{N}$ alta) favorece a volatilização, imobilização e denitrificação do $\mathrm{N}$ aplicado na superfície (Doran, 1980). J á a menor cobertura morta proporcionada pela resteva de soja, associada ao fato de ser uma fonte adicional de N, permitiria melhor contato direto do adubo com o solo, favorecendo sua retenção e diminuindo a intensidade dos processos de transformação do N. Kitur et al. (1984), aplicando $84 \mathrm{~kg} \mathrm{ha}^{-1}$ de $\mathrm{N}$-nitrato de amônio à superfície na cultura de milho, mostraram um comportamento mais eficiente do SPC em relação ao SPD, com produtividade de 9,1 e 7,8 t ha-1 e recuperação de $\mathrm{N}$ no grão de 45 e $23 \%$ respectivamente. Esse comportamento foi atribuído à maior imobilização de N em SPD devido à maior atividade microbiana no sol o sob esse sistema.

O presente estudo objetivou quantificar as perdas por volatilização de $\mathrm{N}-\mathrm{NH}_{3}$ das fontes sól idas nitrato de amônio (NA), sulfato de amônio (SA) e uréia (U) e das fontes fluidas uran (UR, nitrato deamônio + uréia) e sulfuran (SU, nitrato de amônio + uréia + sulfato de amônio) aplicadas à superfície e incorporadas (5 a $7 \mathrm{~cm}$ ), na cobertura nitrogenada de milho, em dose única de cerca de $100 \mathrm{~kg} \mathrm{ha}^{-1}$ de N no SPD e no SPC.

\section{MATERIAL E MÉTODOS}

O estudo constou de dois experimentos no ano agrícola 1994/95 em SPD, com irrigação suplementar, no Centro de Pesquisa Novartis-Seeds, Rodovia BR 452, km 142 do município de Uberlândia (MG), e em SPC, sequeiro, na Fazenda Sta. Teresinha, Rodovia BR 050, km 102 do mesmo município.

O experimento em SPD foi efetuado em latossolo vermel ho-escuro distrófico argiloso, apresentando $\mathrm{pH}\left(\mathrm{H}_{2} \mathrm{O}\right)$ de 6,$2 ; 32 \mathrm{mg} \mathrm{kg}^{-1}$ de $\mathrm{P}$ (Mehlich); $70 \mathrm{mmol}_{\mathrm{C}} \mathrm{dm}^{-3}$ de CTC; $76 \%$ de saturação de bases; $32 \mathrm{~g} \mathrm{~kg}^{-1}$ de M.O. e 450, 350 e $200 \mathrm{~g} \mathrm{~kg}^{-1}$, respectivamente, deargila, silteeareia, para a camada de $0-20 \mathrm{~cm}$ de profundidade. $O$ experimento em SPC foi realizado em latossolo vermel ho-amarelo, distrófico, arenoso, com $\mathrm{pH}\left(\mathrm{H}_{2} \mathrm{O}\right)$ de 5,$8 ; 7 \mathrm{mg} \mathrm{kg}^{-1}$ de $\mathrm{P}$ (Mehlich); $27,3 \mathrm{mmol}_{\mathrm{C}} \mathrm{dm}^{-3}$ de CTC; $61 \%$ de saturação de bases; $22 \mathrm{~g} \mathrm{~kg}^{-1}$ de M.O. e 140 por 50 e $810 \mathrm{~g} \mathrm{~kg}^{-1}$ de argila, 
siltee areia respectivamente, para a profundidade de $0-20 \mathrm{~cm}$.

NoSPD, o plantio do milho híbrido, triplo, Densus, foi efetuado sobre uma resteva de $5,8 \mathrm{t} \mathrm{ha} \mathrm{-}^{-1}$ de aveiapreta. A adubação de plantio foi de $440 \mathrm{~kg} \mathrm{ha}^{-1}$ do formulado 10-20-25 $\left(\mathrm{N}, \mathrm{P}_{2} \mathrm{O}_{5}, \mathrm{~K}_{2} \mathrm{O}\right)+40 \mathrm{~kg} \mathrm{ha}^{-1}$ de F.T.E.BR-12. NoSPC, o sol o foi subsol ado e gradeado. O plantio do híbrido Densus foi sobre uma área cultivada com soja no verão anterior. A adubação de plantio foi de $400 \mathrm{~kg}$ ha-1 do formulado $10-30-10+$ $50 \mathrm{~kg}$ ha-1 de F.T.E. BR-12, seguida 18 dias após, de uma cobertura de $67 \mathrm{~kg} \mathrm{ha}^{-1}$ de $\mathrm{K}_{2} \mathrm{O}$. Tanto no SPD como noSPC, quatro semanas após o plantio, efetuouse desbaste manual, visando obter um estande médio de 62.500 plantas ha-1. O delineamento experimental, nos dois experimentos, foi de blocos casualizados, com dez tratamentos e duas repetições. As parcel as foram constituídas de quatro linhas, espaçadas de $0,8 \mathrm{~m}$ e $25 \mathrm{~m}$ de comprimento. Os tratamentos de cobertura nitrogenada consistiram em cinco fontes, aplicadas de forma mecanizada na maioria dos tratamentos, aos 33 dias após o plantio (d.a.p.) em dose única de cerca de $100 \mathrm{~kg} \mathrm{ha}^{-1}$ em faixa superficial e enterradas a 5$7 \mathrm{~cm}$ no meio das entrelinhas. As fontes sólidas utilizadas foram nitrato de amônio (NA, $34 \% \mathrm{~N}$ ); sulfato de amônio $(S A, 20,6 \% N)$ e uréia $(U, 45 \% N)$ e, as fluidas, uran (UR, 32\% N-nitrato deamônio +uréia) e sulfuran (SU, 20\% N-nitrato de amônio + uréia + sulfato de amônio). No quadro 1, encontramse as doses médias de $\mathrm{N}$ aplicadas no solo sob os coletores deamônia das cinco fontes noSPD enoSPC. Os valores indicados foram obtidos com base na calibração da adubadeira e do diâmetro do coletor. Com exceção do sulfuran no SPC, as doses aplicadas apresentaram uma variação de apenas $5 \%$ (valor médio de $100,8 \mathrm{~kg} \mathrm{ha}^{-1}$ de $\mathrm{N}$ aplicado). No caso específico do tratamento com sulfuran, no SPC, houve um erro na velocidade da aplicação do trator, o que provocou uma superdosagem. Em ambos os sistemas, a aplicação superficial dos adubos sólidos foi manual, na área de influência dos sistemas coletores de amônia, para obter uma maior precisão na quantidade aplicada. Essecuidado nãofoi necessário com as fontes fluidas, visto o contato mais uniforme, quando aplicadas à superfície. Não houve testemunha sem adubação de cobertura.

No SPD, a área experimental foi irrigada previamenteao plantio com $30 \mathrm{mme}$, posteriormente, efetuaram-se irrigações suplementares de $15 \mathrm{~mm}$, a cada três-quatro dias, em setembro e outubro, até regularização das chuvas. Medidas de pluviometria e temperaturas médias, máximas e mínimas foram realizadas durante o período experimental nos dois locais (Quadro 2).

As perdas gasosas de $\mathrm{N}-\mathrm{NH}_{3}$ foram quantificadas em col etor semi-aberto estático adaptado de Nömmik (1973) por Lara Cabezas \& Trivel in (1990) e calibrado por Lara Cabezas et al. (1997) para estimar perdas reais em condições de campo. A instalação dos col etores foi efetuada em del ineamento inteiramente casualizado, em triplicata, em cima do sulco de adubação de cada tratamento, de tal forma que, em cada experimento foram utilizados trinta coletores (cinco fontes $x$ dois métodos de aplicação $x$ três repetições). Efetuaram-se seis col etas do $\mathrm{N}-\mathrm{NH}_{3}$ volatilizado do absorvedor inferior em intervalos de quatro a cinco dias. Os discos absorvedores foram guardados em saco plástico e armazenados em geladeira $\left(5^{\circ} \mathrm{C}\right)$ para posterior extração da amônia retida. O N-NH 3 retido no disco (NVa) foi extraído e determinado de acordo com Lara Cabezas et al . (1997). Os resultados foram expressos em $\mathrm{mg} \mathrm{m}^{-2}$ de N-NH e transformados para percen-tagem do $\mathrm{N}$ aplicado.

Aos 65 dias após o plantio, em ambas as áreas experimentais, foi col hida, de cada parcela, nas duas

Quadro 1. Doses de N aplicadas sob os coletores de amônia das cinco fontes nitrogenadas na cultura de milho no sistema de plantio direto (SPD) e no sistema de plantio convencional (SPC)(1)

\begin{tabular}{|c|c|c|c|c|c|}
\hline & & \multicolumn{4}{|c|}{ Aplicação de N } \\
\hline \multicolumn{2}{|c|}{ Tratamentos } & \multicolumn{2}{|c|}{ SPD } & \multicolumn{2}{|c|}{ SPC } \\
\hline Fontes $(2)$ & Forma de aplicação & Efetiva & Por ha & E fetiva & Por ha \\
\hline & & $\mathrm{mg} \mathrm{m}^{-2}$ & $\mathrm{~kg} \mathrm{ha}^{-1}$ & $\mathrm{mg} \mathrm{m}^{-2}$ & $\mathrm{~kg} \mathrm{ha}^{-1}$ \\
\hline SA & $\begin{array}{l}\text { Superficial } \\
\text { Incorporada }\end{array}$ & $\begin{array}{l}9.968 \\
9.004\end{array}$ & $\begin{array}{l}99,7 \\
90,0\end{array}$ & $\begin{array}{r}10.008 \\
9.931\end{array}$ & $\begin{array}{r}100,1 \\
99,3\end{array}$ \\
\hline NA & $\begin{array}{l}\text { Superficial } \\
\text { Incorporada }\end{array}$ & $\begin{array}{l}10.552 \\
10.330\end{array}$ & $\begin{array}{l}105,6 \\
103,3\end{array}$ & $\begin{array}{r}9.931 \\
11.695\end{array}$ & $\begin{array}{r}99,3 \\
117,0\end{array}$ \\
\hline$U$ & $\begin{array}{l}\text { Superficial } \\
\text { Incorporada }\end{array}$ & $\begin{array}{l}10.079 \\
10.195\end{array}$ & $\begin{array}{l}100,8 \\
102,0\end{array}$ & $\begin{array}{r}10.173 \\
9.621\end{array}$ & $\begin{array}{r}101,7 \\
96,2\end{array}$ \\
\hline UR & $\begin{array}{l}\text { Superficial } \\
\text { Incorporada }\end{array}$ & $\begin{array}{l}10.233 \\
10.026\end{array}$ & $\begin{array}{l}102,2 \\
100,3\end{array}$ & $\begin{array}{l}9.876 \\
9.888\end{array}$ & $\begin{array}{l}98,8 \\
98,9\end{array}$ \\
\hline SU & $\begin{array}{l}\text { Superficial } \\
\text { Incorporada }\end{array}$ & $\begin{array}{r}10.000 \\
9.991\end{array}$ & $\begin{array}{r}100,0 \\
99,9\end{array}$ & $\begin{array}{l}18.322 \\
18.328\end{array}$ & $\begin{array}{l}183,2 \\
183,3\end{array}$ \\
\hline
\end{tabular}

(1) Médias de três coletores. ${ }^{(2)}$ SA: sulfato de amônio; NA: nitrato de amônio; U: uréia; UR: uran (nitrato de amônio + uréia); SU: sulfuran (nitrato de amônio + uréia + sulfato de amônio). 
Quadro 2. Registro de parâmetros climáticos no Centro de Pesquisa Novartis-Seeds (SPD) e na Fazenda Sta. Teresinha (SPC) durante o período experimental(1)

\begin{tabular}{|c|c|c|c|c|c|c|c|}
\hline \multirow{2}{*}{ Parâmetro } & \multicolumn{7}{|c|}{ Meses (1994-95) } \\
\hline & Setembro & Outubro & Novembro & Dezembro & J aneiro & Fevereiro & Março \\
\hline & \multicolumn{7}{|c|}{ Centro de Pesquisa Ciba-Sementes (SPD) } \\
\hline Pluviometria, mm & 0 & 141 & 129 & 264 & 127 & $\ldots$ & $\ldots$ \\
\hline Irrigação, mm & 135 & 60 & 0 & 0 & 0 & $\ldots$ & $\ldots$ \\
\hline Temperatura, ${ }^{\circ} \mathrm{C}$ Máx. & 34 & 38 & 36 & 35 & 32 & $\ldots$ & $\ldots$ \\
\hline \multirow[t]{2}{*}{ Temperatura, ${ }^{\circ} \mathrm{C}$ Mín. } & 17 & 19 & 19 & 20 & 20 & $\ldots$ & $\ldots$ \\
\hline & \multicolumn{7}{|c|}{ Fazenda Sta. Teresinha (SPC) } \\
\hline Pluviometria, mm & $\ldots$ & $\ldots$ & 32 & 381 & 255 & 394 & 103 \\
\hline Temperatura, ${ }^{\circ} \mathrm{C}$ Máx. & $\ldots$ & $\ldots$ & 26 & 29 & 29 & 28 & 29 \\
\hline Temperatura, ${ }^{\circ} \mathrm{C}$ Mín. & $\ldots$ & $\ldots$ & 18 & 19 & 19 & 18 & 18 \\
\hline
\end{tabular}

${ }^{(1)}$ No SPD (sistema de plantio direto) os registros foram de 16/9/94 (plantio) até 23/1/95 (col heita). No SPC (sistema de plantio convencional) de 22/11/94 (plantio) até 1/4/95 (col heita).

linhas centrais, a parte aérea de cinco plantas por parcela, para determinação de massa seca e quantidade de $\mathrm{N}$ acumulado. $\mathrm{Na}$ colheita, determinaram-se o número de plantas, o peso de 1.000 grãos, a produção de grãos (umidade corrigida para $130 \mathrm{~g} \mathrm{~kg}^{-1}$ ) e a massa seca de grão, que foi colocado em estufa $\left(60^{\circ} \mathrm{C}\right)$ até peso constante para a determinação do $\mathrm{N}$ exportado para o grão. $\mathrm{O} \mathrm{N}$ foi determinado por digestão sulfúrica e destilação Kjel dahl pelo método de Parkinson \& Allen (1975).

$O$ efeito das fontes e formas de aplicação na volatilização de amônia, nos parâmetros defitomassa e absorção de $\mathrm{N}$ pela cultura em cada sistema de plantio, foi avaliado por análise da variância em esquema fatorial $5 \times 2$, e as médias dos tratamentos, comparadas pelo teste de Tukey ao nível de $5 \%$. Os parâmetros de massa seca e $\mathrm{N}$ acumulado na etapa deflorescimento e de peso de 1.000 grãos, $\mathrm{N}$ exportado para o grão e produtividade foram avaliados deforma similar.

\section{RESULTADOS E DISCUSSÃO}

No quadro 3 são apresentadas perdas acumuladas de $\mathrm{N}-\mathrm{NH}_{3}$ volatilizado das cinco fontes nitrogenadas, 24 e 26 dias após aplicação no SPD e SPC respectivamente. No SPD, as perdas mais significativas foram das fontes aplicadas na superfície dos resíduos. Em ordem decrescente foram de 78,0; 37,$2 ; 26,9 ; 10,2$ e 7,9\% do N aplicado das fontes uréia, uran, sulfuran, sulfato de amônio enitrato deamônio respectivamente. As perdas apresentaram relação direta com a presença de $\mathrm{N}$-amídico nas fontes: $45 \%$ para a uréia, $14 \%$ para o uran e $8 \%$ para o sulfuran. Segundo Bremmer (1995), o risco de perdas gasosas por vol atilização de amônia é particularmenteal to em sistemas reduzidos de manejo agrícolas, nos quais a uréia é aplicada superficialmente. A maior atividade da urease da palhada em relação ao solo (E ckert et al., 1986) deve facilitar a rápida hidrólise do N-amídico, favorecendo sua volatilização. De fato, as perdas do $\mathrm{N}$-uréia registradas atéas 192 horas após a aplicação foram de 50,8\% do N aplicado (Figura 1a).

Por outro lado, dois dias antes da adubação de cobertura nitrogenada, registraram-se $31 \mathrm{~mm}$ de precipitação, o que deve ter contribuído para a manutenção da umidade na palhada, facilitando a dissolução rápida da uréia. Decorridos oito dias da adubação, houve três eventos pluviométricos, de 4, 6 e $13 \mathrm{~mm}$ de água, aos 3, 5 e 7 dias respectivamente, os quais não devem, provavel mente, ter sido suficientes para lavar a uréia da pal hada à superfície. As perdas expressivas do $\mathrm{N}$-uran incorporado $(28,1 \%$ do N aplicado, valor não considerado na comparação estatística) ocorreram por um erro na forma de aplicação. No SPC, as perdas foram de 30,7; 18,3; 9,8; 3,7 e 2,5\% do $\mathrm{N}$ aplicado na superfície das fontes uréia, sulfuran, uran, sulfato de amônio enitrato de amônio respectivamente(Quadro 3). A perda registrada pelo sulfuran foi na base de uma aplicação de $183 \mathrm{~kg}$ ha-1 de N (Quadro 1). Em dosagens elevadas, a volatilização de amônia apresenta um comportamento exponencial (Overrein \& Moe, 1967; Rodrigues \& Kiehl, 1986). As maiores perdas de $\mathrm{N}-\mathrm{NH}_{3}$ do tratamento sulfuran, proporcionais ao conteúdo de $\mathrm{N}$ amídico, podem ser atribuídas à maior dosagem de $\mathrm{N}$ aplicado. Na figura $1 \mathrm{lb}$ são apresentadas as perdas de $\mathrm{N}-\mathrm{NH}_{3}$ no decorrer do período de amostragem, sendo registradas para a uréia, na superfície, perdas acumuladas de 22,2\% do N, 192 horas após aplicação. Dois dias antes da aplicação da cobertura nitrogenada, foram registrados $4 \mathrm{~mm}$ de chuva, e, depois, $44 \mathrm{~mm}$ de chuva, os quais devem ter contribuído para a incorporação da uréia no sol o. Esses resultados estão indicando que é mais importante o umedecimento do 
Quadro 3. Perdas acumuladas de $\mathbf{N}-\mathrm{NH}_{3}$ volatilizado provenientes da aplicação superficial e incorporada de cinco fontes nitrogenadas à cobertura de milho nos dois sistemas de plantio(1)

\begin{tabular}{|c|c|c|c|c|c|}
\hline \multirow{3}{*}{ Fonte nitrogenada } & \multicolumn{4}{|c|}{ Forma de aplicação } & Média \\
\hline & \multicolumn{2}{|c|}{ Superficial } & \multicolumn{3}{|c|}{ Incorporada } \\
\hline & $\mathrm{mg} \mathrm{m}^{-2}$ & $\%$ do $\mathrm{N}$ aplicado & $\mathrm{mg} \mathrm{m}^{-2}$ & $-\% c$ & cado - \\
\hline & \multicolumn{5}{|c|}{ Sistema de plantio direto } \\
\hline Sulfato de amônio & 1.018 & $10,2 a$ & 193 & $2,1 a$ & $6,2 \mathrm{~A}$ \\
\hline Nitrato de amônio & 833 & $7,9 a$ & 177 & $1,7 \mathrm{a}$ & $4,8 \mathrm{~A}$ \\
\hline Uréia & 7.849 & $78,0 \mathrm{c}$ & 213 & $2,1 a$ & $40,1 \mathrm{C}$ \\
\hline Uran & 3.810 & $37,2 b$ & 2.824 & - & $37,2 \mathrm{C}$ \\
\hline Sulfuran & 2.688 & $26,9 b$ & 205 & $2,0 a$ & $14,5 \mathrm{~B}$ \\
\hline $\begin{array}{l}\text { Média } \\
\text { dms fontes } x \text { modo (Tukey, } 0,05 \text { ) }\end{array}$ & - & $2,0 \mathrm{~A}$ & - & $2,0 \mathrm{~B}$ & \\
\hline Dentro de modo superficial & & & & & 12,9 \\
\hline Dentro de modo incorporato & & & & & 12,0 \\
\hline \multirow[t]{2}{*}{ C.V. (\%) } & & & & & 27,8 \\
\hline & \multicolumn{5}{|c|}{ Sistema de plantio convencional } \\
\hline Sulfato de amônio & 370 & $3,7 a$ & 195 & $2,0 a$ & $2,8 \mathrm{AB}$ \\
\hline Nitrato de amônio & 263 & $2,5 a$ & 175 & $1,5 a$ & $2,0 \mathrm{~A}$ \\
\hline Uréia & 3.110 & $30,7 d$ & 417 & $4,3 a$ & $17,5 \mathrm{D}$ \\
\hline Uran & 961 & $9,8 b$ & 255 & $2,6 a$ & $6,2 B$ \\
\hline Sulfuran & 3.346 & $18,3 \mathrm{c}$ & 617 & $3,4 a$ & $10,8 \mathrm{C}$ \\
\hline Média & & $13,0 \mathrm{~A}$ & - & $2,8 \mathrm{~B}$ & \\
\hline dms fontes $x$ modo (Tukey, 0,05 ) & & & & & 5,8 \\
\hline C.V. (\%) & & & & & 29,0 \\
\hline
\end{tabular}

(1) As médias entre fontes (coluna) e entre modos de aplicação (linha), seguidas de letras maiúsculas desiguais, diferem estatisticamente pelo teste de Tukey ao nível de $5 \%$. As médias das fontes dentro de cada forma de aplicação (colunas), seguidas de letras minúsculas iguais, não diferem estatisticamente pelo teste de Tukey ao nível de 5\%.

a)

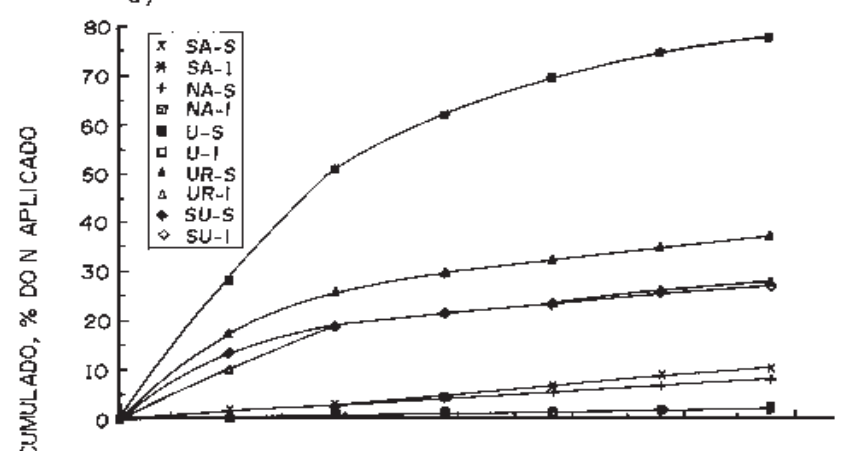

b)

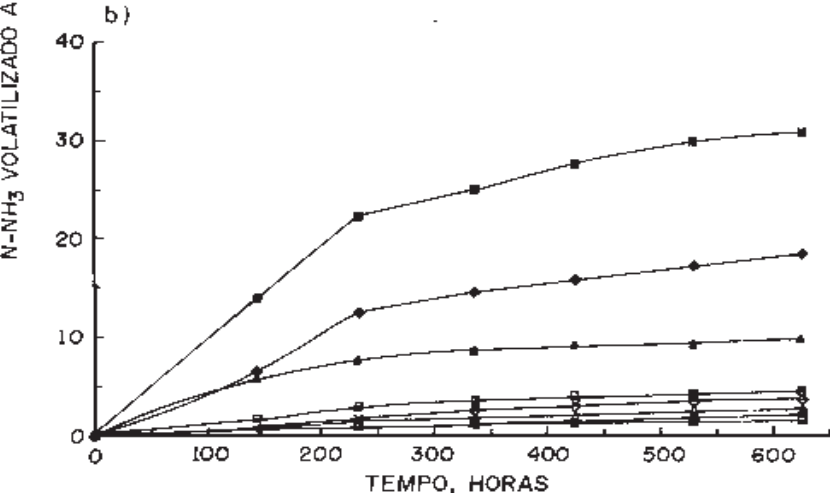

Figura 1. Perdas acumuladas de $\mathrm{N}-\mathrm{NH}_{3}$, ao longo do tempo, provenientes da aplicação superficial (S) e incorporada (I ) de uréia (U), nitrato de amônio (NA), sulfato de amônio (SA), uran (UR) e sulfuran (SU) na cobertura nitrogenada de milho. a: SPD; b: SPC. sol o depois da aplicação da uréia na superfície, do que a condição de umedecimento do solo no momento da aplicação. Tanto o sulfato de amônio como o nitrato de amônio registraram as menores perdas gasosas tanto em SPD com em SPC, apesar das diferentes condições experimentais. O quadro 3 mostra, ainda, que independentemente da fonte e do sistema de plantio, a volatilização de $\mathrm{N}-\mathrm{NH} 3$ foi superior quando os fertilizantes foram aplicados na superfície. Entretanto, as diferenças entre superfície e incorporado foram ampliadas no SPD.

$\mathrm{Na}$ etapa de florescimento do milho, foi efetuada, em ambos os locais, a determinação de massa seca e do $\mathrm{N}$ absorvido (Quadro 4).

NoSPD, independentemente da fonte, houve menor produção de massa seca e absorção de $\mathrm{N}$ pela parte aérea, quando as fontes foram incorporadas. A pal hada de aveia-preta apresenta alta relação C/N (Monegat, citado por Sá, 1993); isso poderia ter criado condições de falta de N no solo, favorecendo o processo de imobilização e, conseqüentemente, menor absorção do N pel a cultura (Varsa et al ., 1995; Sá, 1996). Tanto a volatilização quanto a imobilização foram de grande importância, principalmente no caso da uréia. NoSPC, a resteva da soja deve ter fornecido uma fonte adicional de $\mathrm{N}$ à cultura, enquanto as menores perdas de $\mathrm{N}-\mathrm{NH}_{3}$ volatilizado, juntamente com o processo de imobilização comparativamente menor, teriam favorecido uma eficiência superior da uréia, o que estaria refletido tanto na produção de massa seca como na absorção do N pela planta. 
Quadro 4. Massa seca e N absorvido pela parte aérea do milho na época do florescimento nos dois sistemas de plantio

\begin{tabular}{|c|c|c|c|c|c|c|}
\hline \multirow{2}{*}{$\begin{array}{c}\text { Fonte } \\
\text { nitrogenada }\end{array}$} & \multicolumn{2}{|c|}{ Massa seca } & \multicolumn{2}{|c|}{ Teor de $\mathbf{N}$} & \multicolumn{2}{|c|}{$\mathbf{N}$ acumulado } \\
\hline & Superficial & Incorporado & Superficial & Incorporado & Superficial & Incorporado \\
\hline & \multicolumn{2}{|c|}{$\mathrm{kg} \mathrm{ha}^{-1} \longrightarrow$} & \multicolumn{2}{|c|}{$\mathrm{g} \mathrm{kg}^{-1} \longrightarrow$} & \multicolumn{2}{|c|}{$\mathrm{kg} \mathrm{ha}^{-1}$} \\
\hline & \multicolumn{6}{|c|}{ Sistema de plantio direto } \\
\hline Sulfato de amônio & 8.770 & 8.326 & 17,4 & 14,9 & 153 & 124 \\
\hline Nitrato de amônio & 7.847 & 6.131 & 18,9 & 17,3 & 148 & 106 \\
\hline U réia & 6.070 & 5.907 & 17,7 & 16,1 & 107 & 95 \\
\hline Uran & 8.668 & 7.420 & 16,1 & 17,0 & 140 & 126 \\
\hline Sulfuran & 9.242 & 7.920 & 17,1 & 16,4 & 148 & 130 \\
\hline Média & 8.119 & 7.141 & 17,4 & 16,3 & 141 & 116 \\
\hline \multicolumn{7}{|l|}{ Entre fontes } \\
\hline (DMS, Tukey, 0,05) & \multirow{2}{*}{\multicolumn{2}{|c|}{3.495 (ns) }} & - & - & \multicolumn{2}{|c|}{60 (ns) } \\
\hline \multicolumn{5}{|c|}{ Entre modos de aplicação } & & \\
\hline (DMS, Tukey, 0,05) & \multicolumn{2}{|c|}{1.486 (ns) } & - & - & \multicolumn{2}{|c|}{25 (ns) } \\
\hline \multirow[t]{2}{*}{ C.V. (\%) } & \multicolumn{2}{|c|}{19,3} & - & - & \multicolumn{2}{|c|}{19,5} \\
\hline & \multicolumn{6}{|c|}{ Sistema de plantio convencional } \\
\hline Sulfato de amônio & 11.697 & 10.731 & 16,6 & 14,6 & 194 & 157 \\
\hline Nitrato de amônio & 9.386 & 10.021 & 15,2 & 13,4 & 143 & 134 \\
\hline U réia & 11.404 & 12.064 & 15,5 & 15,4 & 177 & 186 \\
\hline Uran & 10.125 & 10.806 & 13,1 & 16,9 & 133 & 186 \\
\hline Sulfuran & 10.526 & 10.009 & 15,8 & 14,7 & 166 & 147 \\
\hline Média & 10.628 & 10.326 & 15,2 & 15,0 & 163 & 162 \\
\hline \multicolumn{7}{|l|}{ Entre fontes } \\
\hline (DMS, Tukey, 0,05) & \multicolumn{2}{|c|}{4.830 (ns) } & - & - & \multicolumn{2}{|c|}{80 (ns) } \\
\hline \multicolumn{7}{|l|}{ Entre modos de aplicação } \\
\hline (DMS, Tukey, 0,05) & \multicolumn{2}{|c|}{2.054 (ns) } & - & - & \multicolumn{2}{|c|}{34 (ns) } \\
\hline C.V. (\%) & \multicolumn{2}{|c|}{19,0} & - & - & \multicolumn{2}{|c|}{20,6} \\
\hline
\end{tabular}

No quadro 5 são apresentados os resultados de peso de 1.000 grãos, N exportado pel o grão e produtividade, em ambos os sistemas de plantio. Não houve diferença significativa entre as fontes para os parâmetros avaliados.

De acordo com Anghinoni (1985), outras perdas de N podem, simultaneamente, ocorrer, mascarando e até sobrepondo o efeito das perdas gasosas. Nesse sentido, no SPD, além das perdas por volatilização, pode ter havido imobilização, ainda que temporária.J á noSPC podem ter sucedido perdas por lixiviação (não quantificadas), devido à alta pluviometria acontecida após a adubação, num solo de textura arenosa e de baixa CTC (30 $\left.\mathrm{mmol}_{\mathrm{c}} \mathrm{dm}^{-3}\right)$. A ausência de resposta observada, entre fontes, nem sempre constitui um indicador de eficiência, mesmo porque a comparação estatística de produtividade entre os tratamentos mascara a dinâmica do $\mathrm{N}$ aplicado. Maior atenção deve ser dada aos processos de transformação de $\mathrm{N}$ no solo. Em ambos os sistemas, houve uma média de 13,4 kg deN exportado por tonelada de grão produzi da, muito similar à obtida por Coelho et al. (1992), com uma média de $12,3 \mathrm{~kg}$ de $\mathrm{N}$ por tonel ada de grão, média de oito tratamentos de adubação nitrogenada.

A figura 2 mostra o ajuste efetuado entre a produtividade relativa do milho e as perdas gasosas de $\mathrm{N}-\mathrm{NH}_{3}$ para os dois sistemas de plantio. $\mathrm{Na}$ correlação, foram excluídos os valores de produtividade correspondentes às perdas de $\mathrm{N}-\mathrm{NH}_{3}$ do uran incorporado em SPD e de sulfuran aplicado na superfície e incorporado em SPC. Verifica-se que a correlaçãonegativa está indicando que mai ores perdas deN por volatilização correspondem a menor produção relativa, independentemente do sistema de manejo.

Em SPD, para uma magnitude de perdas de $76,8 \mathrm{~kg} \mathrm{ha}^{-1}$ de N volatilizado (diferença entrea maior perda verificada: $78,6 \mathrm{~kg} \mathrm{ha}^{-1}$ de $\mathrm{N}$, e a menor perda média: $1,8 \mathrm{~kg} \mathrm{ha}^{-1}$ de $\mathrm{N}$ ), foi estimada uma queda de produtividade relativa de $9,8 \%\left(1.025 \mathrm{~kg} \mathrm{ha}^{-1}\right.$ de grãos), correspondendo, portanto, a uma queda de produtividade de $13,3 \mathrm{~kg}$ ha-1 de grãos por quilograma de N volatilizado. Por outro lado, em SPC, a queda de $3,8 \%$ na produtividade relativa representou uma queda de $11,8 \mathrm{~kg} \mathrm{ha}^{-1}$ de grãos por quilograma de $\mathrm{N}$ volatilizado. Esses resultados colocam em evidência a grande dificuldade de associar perdas gasosas à produtividade. Diferentemente do verificado por Lara Cabezas et al. (1997) em que, por quilograma de N volatilizado, a produção foi diminuída em 19,3 kg ha-1 de grãos, neste estudo, os antecedentes discutidos permitem concluir que a maior fertilidade dos solos supriu a cultura com maior quantidade de $\mathrm{N}$-nativo, minimizando o efeito do $\mathrm{N}$ volatilizado na queda de produção. Sá (1993) mostrou, para a média de três 
Quadro 5. Massa de mil grãos e produtividade do milho em SPD e SPC

\begin{tabular}{|c|c|c|c|c|c|c|}
\hline \multirow{2}{*}{$\begin{array}{c}\text { Fonte } \\
\text { nitrogenada }\end{array}$} & \multicolumn{2}{|c|}{ Massa 1.000 grãos } & \multicolumn{2}{|c|}{ N exportado } & \multicolumn{2}{|c|}{ Produtividade } \\
\hline & Superficial & Incorporado & Superficial & Incorporado & Superficial & Incorporado \\
\hline & \multicolumn{2}{|c|}{$\longrightarrow \mathrm{g} \longrightarrow$} & \multicolumn{4}{|c|}{$\mathrm{kg} \mathrm{ha}^{-1}$} \\
\hline & \multicolumn{6}{|c|}{ Sistema de plantio direto } \\
\hline Sulfato de amônio & 350 & 337 & 128 & 134 & 10.042 & 9.256 \\
\hline Nitrato de amônio & 356 & 343 & 131 & 140 & 9.536 & 9.860 \\
\hline Uréia & 335 & 339 & 116 & 132 & 9.151 & 9.413 \\
\hline Uran & 327 & 349 & 118 & 133 & 8.976 & 9.639 \\
\hline Sulfuran & 321 & 354 & 123 & 132 & 8.776 & 10.460 \\
\hline Média & 339 & 344 & 123 & 134 & 9.296 & 9.725 \\
\hline \multicolumn{7}{|l|}{ Entre fontes } \\
\hline (DMS, Tukey, 0,05) & \multicolumn{2}{|c|}{40 (ns) } & 37 (ns) & \multicolumn{3}{|c|}{2.006 (ns) } \\
\hline \multicolumn{7}{|c|}{ Entre modos de aplicação } \\
\hline (DMS, Tukey, 0,05) & \multicolumn{2}{|c|}{17 (ns) } & 16 (ns) & \multicolumn{3}{|c|}{853 (ns) } \\
\hline \multirow[t]{2}{*}{ C.V. (\%) } & \multicolumn{2}{|c|}{4,9} & 12,0 & \multicolumn{3}{|c|}{8,9} \\
\hline & \multicolumn{6}{|c|}{ Sistema de plantio convencional } \\
\hline Sulfato de amônio & 380 & 396 & 129 & 124 & 9.428 & 9.644 \\
\hline Nitrato de amônio & 385 & 369 & 144 & 121 & 9.818 & 9.470 \\
\hline Uréia & 398 & 398 & 132 & 124 & 9.717 & 9.583 \\
\hline Uran & 390 & 385 & 124 & 119 & 9.583 & 9.286 \\
\hline Sulfuran & 394 & 383 & 131 & 130 & 9.812 & 8.947 \\
\hline Média & 389 & 386 & 132 & 124 & 9.672 & 9.386 \\
\hline \multicolumn{7}{|l|}{ Entre fontes } \\
\hline (DMS, Tukey, 0,05) & \multicolumn{2}{|c|}{23 (ns) } & 29 (ns) & \multicolumn{3}{|c|}{2.193 (ns) } \\
\hline \multicolumn{7}{|l|}{ Entre modos de aplicação } \\
\hline (DMS, Tukey, 0,05) & \multicolumn{2}{|c|}{10 (ns) } & 12 (ns) & \multicolumn{3}{|c|}{932 (ns) } \\
\hline C.V. (\%) & \multicolumn{2}{|c|}{2,5} & 9,4 & \multicolumn{3}{|c|}{9,6} \\
\hline
\end{tabular}

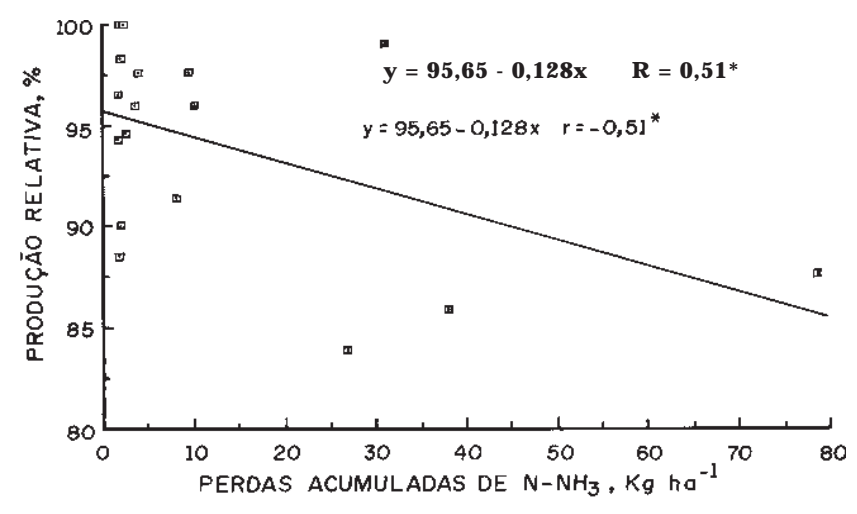

Figura 2. Efeito das perdas gasosas de $\mathbf{N}-\mathbf{N H}_{3}$ volatilizado sobre a produtividade relativa do milho no SPD e SPC.

híbridos de milho em SPD, quando adubados com doses de 60, 120, 180 e 240 kg ha-1 de N em cobertura, uma redução na produção de grãos de 15,0, 12,8, 7,0 e $3,3 \mathrm{~kg} \mathrm{ha}^{-1}$ respectivamente. Cabe destacar os altos val ores de produtividade dos tratamentos-testemunha nesses resultados, como indicador da importância do solo no suprimento de $\mathrm{N}$ à cultura.

\section{CONCLUSÕES}

1. Tanto no SPD como no SPC as perdas por volatilização de $\mathrm{N}-\mathrm{NH}_{3}$ foram expressivas quando aplicadas, respectivamente, sobre a pal hada deaveiapreta e na superfície do solo.

2. O nitrato de amônio e o sulfato de amônio apresentaram perdas inferiores a $15 \%$ do $\mathrm{N}$ aplicado na superfície, em ambos os sistemas de plantio.

3. As diferenças de comportamento entre as fontes em termos de perdas por volatilização não se refletiram sobre a produtividade do milho.

\section{AGRADE CIMENTOS}

Ao Centro de Pesquisa Novartis-Seeds, EmbrapaCNPMS, Sete Lagoas (MG), e I piranga Serrana Fertilizantes, J aú (SP), pela col aboração financeira na realização deste estudo. Aos al unos do Departamento de Agronomia da UFU, Srs. Lício A. Pena de Saire, Fernando N. Faggioni, Alexandre M. Lellis e Marcos G. Bortol etto, pela execução da fase experimental. 


\section{LITERATURA CITADA}

ANGHINONI, I. Adubação nitrogenada nos Estados do Rio Grande do Sul e Santa Catarina. In: SANTANA, M.B.M., ed., Adubação nitrogenada no Brasil. 1.ed. Ilhéus, CEPLAC/ Sociedade Brasileira de Ciência do Solo, 1985. p.1-18.

BOUWMEESTER, R.J .; VLEK, P.L. \& STUMPE, J.M. Effect of environmental factors om ammonia volatilization from ureafertilized soil. Soil Sci. Soc. Am. J ., Madison, 49:376-381, 1985.

BREMMER, J.M. Recent research on problems in the use of urea as a nitrogen fertilizer. Fert. Res., The Netherlands, 42:321329, 1995.

COELHO, A.M.; FRANÇA, G.E.; BAHIA FILHO, A.F.C. \& GUEDES, G.A.A. Doses e métodos de aplicação de fertilizantes nitrogenados na cultura de mil ho sob irrigação. R. bras. Ci. Solo, Campinas, 16:61-67, 1992.

DORAN, J.W. Soil microbial and biochemical changes associated with reduced tillage. Soil Sci. Soc. Am. J., Madison, 44:765771, 1980.

ECKERT, D.J .; DICK, W.A. \& J OHNSON, J.W. Response of notillage grown in corn and soybean residues to several nitrogen fertilizer sources. Agron. J., Madison, 78:231-235, 1986.

ERNST, J.W. \& MASSEY, H.F. The effects of several factors on volatilization of ammonia formed from urea in the soil. Soil Sci. Soc. Am. Proc., Madison, 24:87-90, 1960.

KITUR, B.K.; SMITH, M.S.; BLEVINS, R.L. \& FRYE, W.W. Fate of $15 \mathrm{~N}$-depleted ammonium nitrate applied to no-tillage and conventional tillage corn. Agron. J., Madison, 76:240-242, 1984.

LARA CABEZAS, W.A.R. \& TRIVELIN, P.C.O. Eficiência de um coletor semi-aberto estático na quantificação de $\mathrm{N}-\mathrm{NH}_{3}$ volatilizado da uréia aplicada ao solo. R. bras. Ci. Solo, Campinas, 14:345-352, 1990.

LARA CABEZAS, W.A.R.; KORNDÖRFER, G.H. \& MOTTA, S.A. Volatilização de $\mathrm{N}-\mathrm{NH}_{3}$ na cultura de milho: I Efeito da irrigação esubstituição parcial da uréia por sulfato deamônio. R. bras. Ci. Solo, Viçosa, 21:481-487. 1997b.
MENGEL, D.B.; NELSON, D.W. \& HUBER, D.M. Placement of nitrogen fertilizers for no-till and conventional till corn. Agron. J., Madison, 74:515-518, 1982.

NÖMMIK, H. The effect of pellet size on the ammonia loss from urea applied to forest soil. Plant \& Soil, The Hague, 39:309318, 1973.

OVERREIN, L.N. \& MOE, P.G. Factors affecting urea hydrolysis and ammonia volatilization in soil. Soil Sci. Soc. Am. Proc., Madison, 31:57-61, 1967.

PARKINSON, J.A. \& ALLEN, S.E. A wet oxidation procedure suitable for the determination of nitrogen and mineral nutrients in biological material. Commun. Soil Sci. Plant Anal., 6:1-11, 1975.

RODRIGUES, M.B. \& KIEHL, J.C. Volatilização de amônia após emprego de uréia em diferentes doses e modos de aplicação. R. bras. Ci. Solo, Campinas, 10:37-43, 1986.

SÁ, J.C. Manejo da fertilidade de solo no sistema plantio direto. 1.ed. In: CNPT-EMBRAPA, FUNDACEP-FECOTRIGO, FUNDAÇÃO ABC. Plantio direto no Brasil. Passo Fundo, Aldeia Norte, 1993. p.37-60.

SÁ, J.C. Manejo de nitrogênio na cultura de milho no sistema de plantio direto. 1.ed. Passo Fundo, Aldeia Norte, 1996. 23p.

STECKER, J . Nitrogen sources and placement method for no-till corn. In: NORTH CENTRAL EXTENSION-INDUSTRY SOIL FERTILITY CONFERENCE, 24., St. Louis, Missouri, 1994. Proceedings. St. Louis, Missouri, Potash \& Phosphate Institute, 1995. p.18-19.

TIMMONS, D.R. \& BAKER, J.L. Fertilizer management effect on recovery of labeled nitrogen by continuous no-till. Agron. J ., Madison, 84:490-496, 1992.

VARSA, E.C.; KELLER, K.E.; J EMISON, J.M.; OSBORN, M.W.; LEIS, A.K. \& HNETKOVSKY, S.W. Nitrogen placement in no-till corn. In: PROCEEDINGS OF NORTH CENTRAL EXTENSION-INDUSTRY SOIL FERTILITY CONFERENCE, 24., St. Louis, Missouri, 1994. Proceedings. St. Louis, Missouri, Potash \& Phosphate Institute, 1995. p.69-74.

VOLK, G.M. Volatile loss of ammonia foll owing surface application of urea to turf or bare soils. Agron. J., Madison, 51:746-749, 1959. 